\title{
Haemophilia: a race for cure
}

Keywords: adenovirus-associated virus, immunosuppression, hepatocellular, BMN 270, FIX-padua, transgenes

\section{Introduction}

Successful haemostasis depends upon complex interactions between cellular and protein components of the blood. ${ }^{1}$ Haemophilia A and haemophilia B, caused by deficiencies of protein coagulation factor VIII (FVIII) and factor IX (FIX) respectively, ${ }^{2}$ result in impaired thrombin generation, unstable clots and spontaneous bleeding in affected individuals. The severity of bleeding is largely proportional to the degree of factor deficiency. ${ }^{2}$ Those with little or no circulating factor $(<1 \%$ of normal activity within the blood) generally experience the most severe symptoms. The syndrome is characterised by spontaneous bleeding ${ }^{2}$ commonly affecting the musculoskeletal

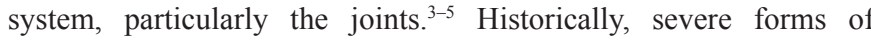
haemophilia carried a very poor prognosis. Fortunately advances in pharmacotherapy and clinical care have improved both morbidity and mortality. ${ }^{6,7}$ The mainstay of treatment continues to be replacement of the missing factor by infusion of FVIII and FIX concentrates, either on demand in response to bleeding episodes or as prophylaxis.

However, despite advances in treatment, patients with severe haemophilia continue to have unmet medical needs. Life expectancy remains lower in men with haemophilia compared to the general male population in the UK (adjusted for $\mathrm{HIV}, \mathrm{HCV}$ infection). ${ }^{7}$ Intracranial hemorrhage is the most common cause of death ${ }^{7}$ and any bleeding leads to pain, impacts upon joint health and quality of life. The success of prophylactic treatments in preventing bleeds depends upon strict adherence to a regime ${ }^{9}$ and as factor is administered intravenously this can be onerous for the patient. Venous access is not always straightforward or comfortable for patients, contributing to poor adherence and failure of treatment. ${ }^{10}$ In addition factor treatment of haemophilia, particularly severe forms, is expensive and it is currently estimated that $70 \%$ of the world's haemophilia population have little or no access to therapy. ${ }^{11}$ Finding economic, well tolerated, yet maximally effective treatments for all patients with severe haemophilia remains a priority.

\section{Gene therapy}

Gene therapy may present an opportunity to cure haemophilia and provide an ultimately acceptable treatment for patients. Although initial treatment might be costly, successful gene therapy would reduce the expense of treatment over a life-time, both in terms of pharmacological support and health infrastructure required to support patients. To this end a number of approaches have been taken in pursuit of this goal. The fundamental aim of gene therapy is to deliver the therapeutic transgene to patient cells, resulting in effective transduction and therapeutic levels of gene expression. The most successful studies in haemophilia have used viral vectors to deliver trans genes. Of the viral vectors used, adenovirus-associated virus (AAV) protocols, using a variety of AAV serotypes, have been favoured in the most recent studies. ${ }^{12-16}$ Wild type AAV produces asymptomatic infections in human. Prior to use in the studies outlined below, modifications are made to eliminate the risk of viral replication. Initial studies in haemophilia B delivered the transgene to
Volume 4 Issue 5 - 2017

\author{
Rea CJ,' Aledort L,' Rangarajan S² \\ 'Business Analyst, USA \\ ${ }^{2}$ Haemophilia Haemostasis \& Thrombosis Centre, UK
}

Correspondence: Catherine Rea, Senior Business Analyst, Massachusetts, USA, Email catherine.rea I@nhs.net

Received: August 31, 2016 | Published: May 15, 2017

skeletal muscles and demonstrated transient expression. ${ }^{17}$ However, the most recent studies and on-going studies, outlined below, have achieved more sustained expression by inducing hepatocytes to express coagulation factors and there are now a number of candidates in the race to find a cure for haemophilia.

\section{Key findings from gene therapy trials}

The most advanced study to date has used AAV8 as a vector to mediate liver directed gene transfer. ${ }^{14}$ AAV8 was selected due to the low frequency of acquired immunity in the population. The Phase 1 study recruited 6 participants with severe haemophilia B to a dose escalation study. 2 participants per cohort received infusions containing low, medium or high doses of AAV8. Sustained expression of FIX, between 1 and 5\%, was achieved in all 6 subjects at 4years. 3 participants were able to stop prophylaxis. 3 participants remained on prophylaxis but required less frequent dosing of FIX concentrate to effectively prevent bleeding. Despite these promising results, the initial study identified potential problems. In one participant receiving the high dose of vector, asymptomatic elevation of serum amino transferase was measured. This was associated with the detection of AAV8-capsid-specific T cells in the peripheral blood. A second individual in the cohort had an isolated increase in liver-enzyme levels. The postulated mechanism has been a cellular immune response to the AAV8 capsid. Treatment with systemic steroids normalised the liver enzymes, but prompted an extension trial to further evaluate the effects of high doses of AAV8. ${ }^{13}$ The 4 further patients recruited to receive high-dose AAV8 infusion all achieved FIX expression. However, 2 had elevated liver enzymes, associated with variable degrees of loss of gene expression.

In summary, all 10patients recruited have expressed FIX, with the higher dose cohort achieving higher levels of factor expression. The effect has been maintained for between 1 and 5years post treatment. No participant has developed inhibitory FIX antibodies nor have late adverse effects been reported, but there has been evidence of an immune mediated process leading to hepatocellular toxicity and variable loss of gene expression. ${ }^{13,14}$ Developing from this data a number of further projects have sought to refine the technology, aiming to improve levels of gene expression, longevity of response whilst eliminating adverse effects. To enhance the efficacy FIX expression AAV8 vectors have been modified to carry a naturally occurring, highly active FIX gene variant, FIX-Padua. ${ }^{16,18}$ In the second highest dosing cohort all participants have expressed the FIX gene and achieved detectable FIX levels 1 has had sustained FIX expression levels of 20-25 percent for 
12 months. In data released to date, 7 patients have been recruited to receive escalating doses of the product BAX355, at three dosing levels. The 2patients in the lowest dosing cohort have achieved low-level gene expression 1 of 2patients achieved FIX expression in the lowest dosing cohort and this has been sustained at 2-3\% activity at months and allowed discontinuation of prophylaxis with factor concentrate. In the second dosing cohort all participants have expressed the FIX gene and achieved detectable FIX levels with 1 sustaining FIX expression levels of $20-25 \%$ for 12 months. 2 others lost their response within 4weeks. This was not associated with evidence of liver inflammation, T cell activation in response to vector or the development of inhibitory inhibitors antibodies to factor IX or to FIX-Padua.

In the highest dose cohort, expression levels have peaked above $50 \%$. However, as with the previously described study, the two patients in this cohort experienced an immune response, variable transaminitis and loss of expression. No FIX inhibitors have been detected and recruitment is to continue in this study. ${ }^{16}$ In parallel, a study using AAV5 as a vector to deliver FIX is in Phase I/II clinical trial. 5patients with severe or moderate haemophilia B (FIX levels less than 2\%), receiving factor prophylaxis have been recruited to a low dose cohort. The first 2 recruited have had factor levels of 4.5 and $5.5 \%$ recorded at 20weeks follow up. 4 of 5 recruited have discontinued prophylaxis. ${ }^{15}$ These studies for gene therapy in haemophilia B are advanced and there are a number of active programmes. By contrast development of effective gene therapy in haemophilia A has, until recently, been lagging. This relates to difficulties in delivering the comparatively large gene and overcoming the immunogenic potential of FVIII. ${ }^{19,20}$ However, recent data from a FVIII gene therapy trial are promising. ${ }^{21}$ To date 8 patients with severe haemophilia A have received a single dose of AAV5 vector carry the FVIII gene (BMN 270). 6patients have been treated at the highest dose and 5 have FVIII levels above 5\% at follow-up ranging from 6-12weeks. 2patients in this cohort have achieved levels above 50\%. In common with the FIX gene trial, a proportion patients have developed transaminitis 2 of the first 3 recruits experienced a raised ALT at between 14 and 28weeks. In both cases this was successfully treated with corticosteroids. 1 affected patient was from the low dose cohort and at no point expressed FVIII above 1\%. The other patient was from the high-dose cohort and to date the FVIII level appears unaffected as he is expressing levels above $50 \%$. All patients subsequently recruited have received prophylactic corticosteroids and no new episodes of transaminitis have been recorded.

\section{Discussion}

Data from the above studies provide encouragement that gene therapy may become a viable treatment. Some patients have expressed factor levels sufficient to convert them from a severe or moderate category of haemophilia $(<5 \%)$ to a mild or even normal phenotype. It this could be achieved in a predictable and sustained fashion then gene therapy could revolutionise the treatment of haemophilia. However, there are clearly still obstacles to overcome. Safety will always be the prime consideration. It is reassuring that the gene therapy products used in the above trials have been well tolerated and no anti-factor antibodies have been reported. However, the numbers of patients recruited to date have been small and mainly within the context of single centre studies. Larger scale and prolonged monitoring will be required prior to widespread clinical implementation of the technology. Long-term safety also needs to considered as there is potential for transgene integration into the host genome and associated insertion or deletion events. A single report of AAV gene transfer inducing hepatocellular carcinoma in a mouse exists. ${ }^{22}$ Other animal studies have been reassuring. ${ }^{23}$ Patients with additional risk factors for cancer (smoking, hepatitis C), may be need to be excluded from gene therapy treatment. In additional, the theoretical increased risks means that treated patients will require long term surveillance. This has associated practical and cost implications and, in combination with the expense of initial treatment, may at least partially negate cost savings from reduced factor usage, putting the therapy beyond the means of some healthcare systems.

The most significant acute toxicity reported in all studies is the occurrence of hepatocellular damage in a number of participants in all the trials reported. ${ }^{15,16,21}$ Cytotoxic T-cells directed against AAV proteins have been identified in association with transaminitis representing an immune mediated process. Fortunately, no sustained injury has been reported and all patients have responded to immune suppression. However, hepatotoxicity and immune activation are clearly associated with a loss of gene expression and a loss of response. If a durable response cannot be expected or predicted, then the considerable initial costs of gene therapy cannot be justified or recouped. To eliminate amnesic responses by memory lymphocytes, patients in each study are excluded if they have antibodies to the chosen vector. However, there may be cross reactivity of antibodies to different AAV serotypes as they can bind in common regions. ${ }^{24}$ As antibodies to AAV are common, screening out those with antibodies to an AAV would preclude treatment of a large proportion of candidates rendering this approach impractical. A future option may be to design out common binding sites from vectors, allowing inclusion of more patients in trials and ultimately allowing gene therapy to be used in a larger population, or consider the use of alternative viral vectors for which there is less pre-existing immunity.

In the BAX355 trial an attempt has been made to reduce product immunogenicity by treating the product to remove "empty capsids" or capsid material not associated with viable viral vector. This is based on laboratory data indicating that cytotoxic $\mathrm{T}$ cell activation to an antigen occurs in a dose dependent fashion. ${ }^{12,24}$ However, this step of removing "empty capsids" in the BAX355 trial has not eliminated immune activation and transaminitis, illustrating the complexity of the immune response and how difficult it will be to overcome. Corticosteroids have been successfully used to suppress immune responses and treat episodes of hepatocellular toxicity. ${ }^{13-16,21}$ There has so far been no data examining the effects of upfront immunosuppression on immune response and the durability of gene therapy treatment. The data from the BMN 270 Trial, where patients are to be prospectively treated with corticosteroids will provide an important assessment of the role that immunosuppression could play in not only reducing occurrence of transaminitis, but also potentially improving the durability and consistency of responses to gene therapy. However, with the introduction of immunosuppression to the regime will necessitate closer monitoring during initiation of treatment and throughout the period of immune suppression therapy. Again this will impact upon the cost, practicality and accessibility of treatment for patients in low income countries. Another concern surrounding lack of treatment durability is that patients will not be able to receive re-treatment with the same gene therapy product. Following gene therapy treatment, patients in trials consistently form antibodies to the vector infused precluding repeat treatments. ${ }^{13,14,16}$ There may be an option to retreat with different products, using alternative vectors (e.g retrovirus), but this possibility will depend upon companies continuing to see the potential benefits of developing and marketing gene therapy products. 


\section{Conclusion}

Long-acting FIX concentrates certainly provide a rival to gene therapy. In low income healthcare systems, reduced frequency of treatment will eventually reduce factor costs. This may allow more patients to receive a degree of prophylaxis, improving clinical outcomes and ultimately reducing burdens on health infrastructures. However, long-acting FVIII products have been less successful in pro-longing half-lives and are unlikely to provide a similar advantage. In addition, neither long-acting nor standard factor products can provide the degree of normalisation in factor level that gene therapy has the potential to deliver. With trough levels occurring between treatments, even patients tightly adhering to prophylaxis regimes will have periods of greater vulnerability to bleeding, whereas successful gene therapy may be able to completely normalise factor levels and fully protect individuals. As a single joint bleed can cause pain and cartilage damage $\mathrm{e}^{3}$ the race for a cure is an aim worth pursuing for all patients with haemophilia.

\section{Acknowledgements}

None.

\section{Conflict of interest}

The author declares no conflict of interest.

\section{References}

1. Hoffman M, Monroe DM. A cell-based model of hemostasis. Thromb Haemost. 2001;85(6):958-965.

2. Maggs PHB, Pasi KJ. Haemophilias A and B. Lancet. 2003;361(9371):1801-1809.

3. Valentino LA. Blood-induced joint disease: the pathophysiology of hemophilic arthropathy. J Thromb Haemost. 2010;8(9):1895-1902.

4. Aledort LM, Haschmeyer RH, Pettersson H. A longitudinal study of orthopaedic outcomes for severe factor-VIII-deficient haemophiliacs. The orthopaedic outcome study group. J Intern Med. 1994;236(4):391399.

5. Hermans C, De Moerloose P, Fischer K, et al. Management of acute haemarthrosis in haemophilia A without inhibitors: literature review, European survey and recommendations. Haemophilia. 2011;17(3):383392.

6. Ikkala E, Helske T, Myllylä G, et al. Changes in the life expectancy of patients with severe haemophilia A in Finland in 1930-79. Br J Haematol. 1982;52(1):7-12.

7. Darby SC, Kan SW, Spooner RJ, et al. Mortality rates, life expectancy, and causes of death in people with hemophilia A or B in the United Kingdom who were not infected with HIV. Blood. 2007;110(3):815-825.

8. Plug I, Peters M, Bunschoten EPM, et al. Social participation of patients with hemophilia in the Netherlands. Blood. 2008;111(4):1811-1815.
9. Collins PW, Björkman S, Fischer K, et al. Factor VIII requirement to maintain a target plasma level in the prophylactic treatment of severe hemophilia A: influences of variance in pharmacokinetics and treatment regimens. J Thromb Haemost. 2010;8(2):269-275.

10. Hacker MR, Geraghty S, Johnson MM. Barriers to compliance with prophylaxis therapy in haemophilia. Haemophilia. 2001;7(4):392-396.

11. O’Mahony B, Black C. Expanding hemophilia care in developing countries. Semin Thromb Hemost . 2005;1(5):561-568.

12. Manno CS, Pierce GF, Arruda VR, et al. Successful transduction of liver in hemophilia by AAV-Factor IX and limitations imposed by the host immune response. Nat Med. 2006;12(3):342-347.

13. Nathwani AC, Reiss UM, Tuddenham EG, et al. Long-term safety and efficacy of factor IX gene therapy in hemophilia B. $N$ Engl J Med. 2014;371(21):1994-2004.

14. Nathwani AC, Tuddenham EG, Rangarajan S, et al. Adenovirusassociated virus vector-mediated gene transfer in hemophilia B. $N$ Engl J Med. 2011;365(25):2357-2365.

15. Uniqure Announces Preliminary Topline Results from Low-Dose Cohort in Hemophilia B Phase I/II Gene Therapy Clinical Trial. USA: MacDougall Biomedical; 2016.

16. Baxalta Reports Continued Progress on Phase 1/2 Clinical Trial of BAX335, Investigational Gene Therapy Treatment for Hemophilia B. USA: Business Wire San Francisco; 2015.

17. Manno CS, Chew AJ, Hutchison S, et al. AAV-mediated factor IX gene transfer to skeletal muscle in patients with severe hemophilia B. Blood. 2003;101(8):2963-2972.

18. Zhang R, Wang Q, Zhang L, et al. Optimized human factor IX expression cassettes for hepatic-directed gene therapy of hemophilia B. Front Med. 2015;9(1):90-99.

19. Swystun LL, Lillicrap D. Gene Therapy for Coagulation Disorders. Circ Res. 2016;118(9):1443-1452.

20. Herzog RW. Hemophilia gene therapy: caught between a cure and an immune response. Mol Ther. 2015;23(9):1411-1412.

21. BioMarin Provides Encouraging Preliminary Data on First 8 Patients in Hemophilia A Gene Therapy Program. USA: BioMarin Pharmaceutical Inc; 2016.

22. Donsante A, Vogler C, Muzyczka N, et al. Observed incidence of tumorigenesis in long-term rodent studies of rAAV vectors. Gene Ther. 2001;8(17):1343-1346.

23. Li H, Malani N, Hamilton SR, et al. Assessing the potential for AAV vector genotoxicity in a murine model. Blood. 2011;117(12):3311-3319.

24. Mingozzi F, High KA. Therapeutic in vivo gene transfer for genetic disease using AAV: progress and challenges. Nat Rev Genet. $2011 ; 12(5): 341-355$. 